\title{
Bona fide colour: DNA prediction of human eye and hair colour from ancient and contemporary skeletal remains
}

Jolanta Draus-Barini ${ }^{1}$, Susan Walsh² ${ }^{2}$ Ewelina Pośpiech ${ }^{4}$, Tomasz Kupiec ${ }^{1}$, Henryk Głąb ${ }^{3}$, Wojciech Branicki ${ }^{1,4^{*}+}$ and Manfred Kayser ${ }^{2+}$

\begin{abstract}
Background: DNA analysis of ancient skeletal remains is invaluable in evolutionary biology for exploring the history of species, including humans. Contemporary human bones and teeth, however, are relevant in forensic DNA analyses that deal with the identification of perpetrators, missing persons, disaster victims or family relationships. They may also provide useful information towards unravelling controversies that surround famous historical individuals. Retrieving information about a deceased person's externally visible characteristics can be informative in both types of DNA analyses. Recently, we demonstrated that human eye and hair colour can be reliably predicted from DNA using the HIrisPlex system. Here we test the feasibility of the novel HIrisPlex system at establishing eye and hair colour of deceased individuals from skeletal remains of various post-mortem time ranges and storage conditions.
\end{abstract}

Methods: Twenty-one teeth between 1 and approximately 800 years of age and 5 contemporary bones were subjected to DNA extraction using standard organic protocol followed by analysis using the HlrisPlex system.

Results: Twenty-three out of 26 bone DNA extracts yielded the full 24 SNP HirisPlex profile, therefore successfully allowing model-based eye and hair colour prediction. HrisPlex analysis of a tooth from the Polish general Władysław Sikorski (1881 to 1943) revealed blue eye colour and blond hair colour, which was positively verified from reliable documentation. The partial profiles collected in the remaining three cases (two contemporary samples and a 14th century sample) were sufficient for eye colour prediction.

Conclusions: Overall, we demonstrate that the HirisPlex system is suitable, sufficiently sensitive and robust to successfully predict eye and hair colour from ancient and contemporary skeletal remains. Our findings, therefore, highlight the HlrisPlex system as a promising tool in future routine forensic casework involving skeletal remains, including ancient DNA studies, for the prediction of eye and hair colour of deceased individuals.

Keywords: Skeletal remains, Forensic DNA phenotyping, Eye colour, Hair colour, HlrisPlex, Ancient DNA, Human appearance, Władysław Sikorski

\footnotetext{
* Correspondence: wbranicki@ies.krakow.pl

${ }^{\dagger}$ Equal contributors

${ }^{1}$ Institute of Forensic Research, Section of Forensic Genetics, Kraków, Poland

${ }^{4}$ Department of Genetics and Evolution, Institute of Zoology, Faculty of

Biology and Earth Sciences, Jagiellonian University, Kraków, Poland

Full list of author information is available at the end of the article
} 


\section{Background}

Skeletal remains represent a unique type of biological material. Due to their unique features, bones and teeth can be resistant to degradation and, depending on the environmental storage conditions, can provide a good source of DNA suitable for genetic analysis thousands of years after an organism's death. DNA analysis of ancient bones and teeth is used to explore various aspects of a species' history, including archaic and modern humans. For instance, the application of next generation DNA sequencing (NGS) technologies to ancient human bones has allowed the establishment of whole genome information on Neanderthals [1] and Denisovans [2]. These studies not only revealed that Denisovans represent a distinct archaic human taxon [2], which was impossible to conclude from the finger bone found as its only evidence, but also highlighted that a genetic admixture likely occurred between both archaic human taxons and modern humans, respectively [1-4].

Contemporary bones and teeth are frequently used in dedicated forensic laboratories dealing with missing person identification cases, including disaster victim identification. Although DNA extraction from skeletal remains is very complex and time consuming with limited possibilities for automation, in forensic science teeth and bones constitute the most suitable sources of DNA for individual identification from heavily decomposed bodies. Forensic DNA analysis of contemporary bones and teeth typically concerns autosomal short tandem repeats (STRs, or microsatellites) for direct match identification with antemortem samples of the same individual, or via STR profile similarities with close putative relatives [5,6]. Mitochondrial (mt) DNA and Y-chromosomal DNA analysis also represent two genetic marker systems that allow profile matching not only with close but also with distant putative maternal and paternal relatives, respectively [7]. Furthermore, there seems to be continual interest in the DNA analysis of remains from famous historical individuals as seen with studies about the last Russian Tsar Nikolaus II (1868 to 1918) and his close family [8-11], the Polish astronomer Nicolaus Copernicus (1473 to 1543) [12], the Italian poet and founder of humanism Francesco Petrarca (1304 to 1374) [13], or perhaps even the evangelist Luke [14] to name but a few examples.

One interesting aspect of ancient and modern skeletal analysis, which has been largely overlooked thus far, is the reconstruction of the deceased person's appearance. In ancient DNA analysis the prediction of particular externally visible characteristics (EVCs) not reflected in skeletal features, such as eye and hair colour, is the only way to get insights into how extinct species may have looked. In contemporary skeletal analysis, such as forensic cases, EVC prediction from DNA can be useful in providing leads in police investigation to reveal the identity of a deceased person if other and more direct means (such as STR, mtDNA and Y-DNA profiling) are non-informative. In recent years, researchers have started seeing increased success in DNA-based EVC prediction, a field sometimes referred to as Forensic DNA Phenotyping (FDP) or DNA intelligence $[15,16]$. From all human externally visible characteristics (besides sex), pigmentation traits, particularly eye and hair colour, are currently the most promising EVCs for DNA prediction. This is due to limited genetic complexity coupled with limited environmental impact that has led to accumulative success in exploring the genetic basis of eye and hair colour via candidate gene and genome-wide association studies [17-22]. From these association studies, highly predictive eye and hair colour DNA markers have been identified [23-33].

Previous ancient DNA studies have suggested that Neanderthals may have had light skin and reddish hair colour due to a particular non-synonymous variation in $M C 1 R$ [34]. Genetic variation in $M C 1 R$ causes light skin colour and red hair colour in modern humans [35], albeit via different DNA variants than found in Neanderthals in that study. Recently, whole genome sequencing (WGS) data from bones of Neanderthals, Denisovans and modern humans from different time periods have been established using NGS technologies and in part have been explored for the prediction of pigmentation (and other visible and non-visible) traits [36-38]. However, the establishment of WGS data from bones, though shown to be feasible via NGS, still comes with a stiff price tag not easily affordable by most laboratories. Furthermore, WGS represents a type of data overkill when one is only interested in specific traits determined by a limited number of genes, such as eye and hair colour. Lastly, WGS comes with the disadvantage that if the overall genome coverage is low, (which often is the case in aDNA NGS/WGS studies), the quality for the particular single nucleotide polymorphisms (SNP) genotypes required for prediction can be low with consequences for the final trait prediction. What, therefore, would be welcomed are more dedicated and targeted approaches allowing the retrieval of high quality EVC information, such as eye and hair colour, from ancient and contemporary human skeletal remains in an effective manner, such as multiplex analyses.

Previously, we developed and forensically validated the IrisPlex system for reliable and accurate DNA prediction of blue and brown eye colour, which includes a sensitive single multiplex assay of six highly eye colour-predictive SNPs and an eye colour prediction model based on genotype and phenotype data of many thousands of individuals from various regions in Europe [24-26]. An independent test dataset of $>3,800$ individuals from seven European sampling sites has shown that the IrisPlex system provides on average $94 \%$ individual-based accuracy for blue and brown eye colour prediction, respectively 
[26]. In an earlier study, some pigmentation associated SNPs had been combined in a multiplex assay and tested successfully on ancient bone samples [39]. However, the predictive value of some of the SNPs considered in that study is low and lower than the SNPs that only became available via subsequent studies. In particular, they typed only two of the six best eye colour predictors included in the IrisPlex assay. Recently, a highly sensitive single multiplex genotyping assay has been developed, together with model-based tools supported by large genotype and phenotype databases, allowing parallel prediction of eye and hair colour from a DNA sample [40]. This so called HIrisPlex system targets 24 eye and/or hair colour predictive DNA polymorphisms (including all 6 IrisPlex SNPs) and provides individual prediction probabilities for eye and hair colour categories as well as hair colour shade categories [40]. As shown recently, this approach allows the prediction of hair colour with average individual-based prediction accuracies of $69.5 \%$ for blond, $78.5 \%$ for brown, $80 \%$ for red and $87.5 \%$ for black hair colour on an independent test set of $>300$ individuals from three European sampling sites [40]. Furthermore, it was previously shown by analysing a worldwide sample set (HGDP-CEPH) that IrisPlex/HIrisPlex eye and hair colour prediction works reliably independent of knowledge on bio-geographic ancestry $[24,40]$. In the present study we test the feasibility of predicting eye and hair colour of deceased individuals from DNA analysis of ancient and contemporary teeth and bones using the novel HIrisPlex system.

\section{Methods}

\section{Bone and teeth samples}

The specimens subjected to DNA analysis were comprised of 21 teeth and 5 bones. Samples S1 to S3 were collected from contemporary human remains approximately one to two years after death as estimated from medico-legal and criminalistic examination. S4 was a tooth collected from modern remains of an incomplete skeleton that rested in soil; it was impossible to estimate an approximate age of the remains but anthropological examination showed they were seriously decayed. Serious degradation was also present in cases S5 to S10, which were contemporary human remains found in a cave. S5 was a fragment of a femur, S6 was a fragment of a tibia, S7 was a fragment of a humerus, S8 was a fragment of another humerus, S9 was a tooth collected from a mandible and S10 was a part of a skull; they were all found in one location in a cave situated in Dolina Będkowska Landscape Park near Kraków. S11 was a tooth collected from the exhumed remains of General Władyslaw Sikorski; the exact date of his death is known (1943) and thus, we know that the analyzed tooth was 69 years of age. S12 to S23 were samples collected from skulls which in 1942 were subjected to bleaching and afterwards kept in a museum in Austria until 1998. S24 was a tooth of a mysterious woman from a Benedictine crypt in Tyniec near Kraków, which according to historical evidence comes from the $12^{\text {th }}$ to $14^{\text {th }}$ century. S25 and S26 were teeth picked up from skeletons found in the St. Andrew Church in Kraków and dated based on historical markers to come from the $14^{\text {th }}$ century.

\section{Precautions taken to avoid contamination}

Multifarious efforts were undertaken in the laboratory in order to prevent contamination. Full protective clothing and separate working localities for extraction, amplification and SBE reaction setup were used. All DNA extractions were done in a separate room reserved for low template DNA samples. DNA extraction and PCR were performed in clean room facilities with Laminar Air Flow benches equipped with HEPA filters (Thermo Fisher Scientific, Hudson, New Hampshire, USA). All working areas and equipment were regularly UV-irradiated and cleaned with bleach. In total, 10 negative extraction controls were included. Each presented case was subjected to separate DNA extraction, except for samples S12 to S23 where three negative extraction controls were used. Negative controls were also included for each PCR amplification performed. All samples were first subjected to STR analysis which showed no traces of DNA admixture that could indicate contamination. Moreover, in order to exclude any possibility of internal contamination, STR (AmpFlSTR ${ }^{\circledR}$ NGM $^{\mathrm{Tw}}$ kit (Applied Biosystems, Foster City, CA, USA)) and HIrisPlex profiles were determined for all 10 members of the laboratory staff. No match was found for any of the samples analysed in both STR and HIrisPlex profiles.

\section{DNA extraction}

DNA was isolated using standard organic extraction protocols. Before extraction, bone material comprising teeth or fragments of femurs was subjected to a purification and decontamination procedure. Samples were treated with $15 \%$ bleach, repeatedly shaken with $70 \%$ ethanol and distilled water, and while drying were subjected to UV irradiation. The entire tooth or bone fragments were then pulverized using FreezerMill 6750 (SPEX CertiPrep Metuchen, New Jersey, USA) and subjected to the extraction procedure. When available, $3 \mathrm{~g}$ (in case of tooth samples 1 to $1.5 \mathrm{~g}$ ) of bone powder were incubated overnight in a water bath set at $56^{\circ} \mathrm{C}$ with $3 \mathrm{~mL}$ of buffer composed of 0.5 M EDTA (Sigma-Aldrich Corporation, St. Louis, Missouri, USA) and 10\% SDS (Amresco LLC, Solon, Ohio, USA) with the addition of $225 \mu \mathrm{L}$ proteinase $\mathrm{K}(10 \mathrm{mg} / \mathrm{mL})$ (Sigma-Aldrich Corporation, St. Louis, Missouri, USA) and $120 \mu \mathrm{L} 1 \mathrm{M}$ DTT (Amresco LLC, Solon, Ohio, USA). The samples were then subjected to double extraction with a phenol-chloroform-isoamyl 
alcohol (Sigma-Aldrich Corporation, St. Louis, Missouri, USA) and concentrated after extraction using Amicon Ultra 4 - 30k columns and Microcon 100 (Millipore, Carrigtwohill, Cork, Ireland) to the final volume of 60 to $70 \mu \mathrm{l}$ in all cases. Negative extraction controls were used to check the purity of the used chemicals and consumables.

\section{DNA quantification}

DNA concentration was assessed on a 7500 Real Time PCR system using Quantifiler ${ }^{\mathrm{TM}}$ Human DNA Quantification Kit (Applied Biosystems, Foster City, CA, USA), following the protocol recommended by the manufacturer.

\section{Genotyping with the HIrisPlex assay}

Multiplex genotyping was performed as described in detail recently [40]. The HIrisPlex assay relies on simultaneous PCR amplification followed by simultaneous SNaPshot primer extension (Applied Biosystems, Foster City, CA, USA) (or minisequencing) of 24 DNA polymorphisms previously established with predictive value on human eye and hair colour, namely N29insA, rs11547464, rs885479, rs1805008, rs1805005, rs1805006, rs1805007, rs1805009, $\mathrm{Y} 152 \mathrm{OCH}, \mathrm{rs} 2228479$, rs1110400 - all from the MC1R gene, rs12913832 from HERC2, rs12203592 from IRF4, rs1042602 and rs1393350 from TYR, rs4959270 from EXOC2, rs28777 and rs16891982 from SLC45A2, rs683 from TYRP1, rs1800407 from OCA2, rs2402130 and 12896399 from SLC24A4, rs12821256 from KITLG and rs2378249 from ASIP. All samples were genotyped at least twice.

\section{Prediction of eye and hair colour with the HIrisPlex system}

Eye colour inference from the genotyping results of the six SNPs (the same as used in IrisPlex) was obtained via model-based prediction using a database of thousands of Europeans and a convenient Microsoft Excel macro (Microsoft Corporation, Redmont, Washington, USA) published previously $[24,26]$. Hair colour inference from the genotypes of 22 SNPs was obtained via model-based prediction using a database of $>1,500$ Europeans with an Excel macro and following a prediction guide that not only considers hair colour categories but also hair colour shade for the final assessment as introduced recently [40].

\section{Results and discussion}

Using a standard organic extraction protocol, we purified genomic DNA from 21 teeth aged between 1 year and approximately 800 years $\left(12^{\text {th }}\right.$ century) and 5 contemporary bones. All samples were subjected to multiple genotyping using the HIrisPlex assay and to modelbased eye and hair colour prediction from the combined HIrisPlex genotypes using large genotype/phenotype databases described elsewhere $[24,26,40]$. It is also worth emphasising that in studies of skeletal remains, unlike some other human characteristics, pigmentation cannot be concluded from basic anthropological research and thus reliable DNA prediction of eye and hair colour shall be particularly useful. The ascertained HIrisPlex genotypes for all 24 DNA polymorphisms and all 26 samples analysed are presented in Table 1 . The derived eye and hair colour probabilities, the predicted eye and hair colours and the accuracy probability values of predicted eye and hair colours for all 26 individuals are provided in Table 2. Although in all but one of the samples analysed the phenotypic eye and hair colour of the deceased individuals analysed were unknown, the previously obtained accuracy estimates from large numbers of individuals with known phenotype and genotype information provide reasonable confidence that the eye and hair colour phenotypes predicted in this study match the true phenotypes of the individuals before death. However, because previous studies showed that the estimated probabilities and attached accuracies are higher for eye than for hair colour categories, more confidence can be expected in eye colour over the hair colour prediction results obtained here.

\section{Contemporary samples}

A number of contemporary forensic DNA identification cases remain unsolved because of no match between the evidence STR profile and a reference DNA profile from a suspect or from the forensic DNA database search. Similarly, a number of missing person cases, including disaster victim identification (DVI) cases, remain unsolved because no STR profile match with the antemortem samples, or because no informative STR profile similarities with known putative relatives can be obtained. In all such cases, EVC information inferred from the scene sample, including skeletal remains, may be valuable for the continuing investigation. Representing typical forensic casework samples, we included three teeth collected from partially or completely skeletonized bodies of approximately one to two years post mortem age. S1 was a cadaver and S2 was a skeleton, both found in small villages in different regions of Poland in the open soil. S3 was a cadaver found inside a building located in Kędzierzyn-Koźle, a small city in southern Poland. The teeth were previously subjected to standard forensic identification using STR and mitochondrial DNA profiling. Complete STR profiles $\left(\mathrm{NGM}^{\mathrm{TM}}\right.$ PCR Amplification Kit or Identifiler ${ }^{\circledR}$ PCR Amplification Kit (Applied Biosystems, Foster City, CA, USA)) were obtained in each case indicating reasonably good preservation of nuclear DNA. The amelogenin gender test revealed that skeletons S1 and S3 were males and S2 a female, corroborating results of the anthropological investigation of the skeletons (data not shown). Complete HIrisPlex profiles were determined for all three samples (Table 1). For S1 to S3 the highest probability $(P)$ was obtained for brown eye colour 
Table 1 HIrisPlex genotyping results together with sample age and starting DNA amount for the 26 samples tested Sample ID Sample/postmortem age Starting template DNA (ng) Genotype

\begin{tabular}{|c|c|c|c|c|c|c|c|c|c|c|c|c|c|c|c|c|c|c|c|c|c|c|c|c|c|c|}
\hline & & & 1 & 2 & 3 & 4 & 5 & 6 & 7 & 8 & 9 & 10 & 11 & 12 & 13 & 14 & 15 & 16 & 17 & 18 & 19 & 20 & 21 & 22 & 23 & 24 \\
\hline S1 & $\mathrm{T} / \mathrm{CR}$ & 0.6 & C & G & C & C & $\mathrm{G} / \mathrm{T}$ & $C$ & $C$ & G & $C$ & G & $\mathrm{T}$ & A & G & A & $C / A$ & $\mathrm{C} / \mathrm{T}$ & G & $G$ & A & $\mathrm{C} / \mathrm{T}$ & $\mathrm{T}$ & $\mathrm{T}$ & C & $\mathrm{G} / \mathrm{T}$ \\
\hline S2 & $\mathrm{T} / \mathrm{CR}$ & 1.7 & C & G & $\mathrm{C} / \mathrm{T}$ & C & $G$ & $C$ & $C$ & $G$ & $C$ & G & $\mathrm{T}$ & A & G & A & $C / A$ & C & G & $\mathrm{G}$ & A & $\mathrm{C} / \mathrm{T}$ & $\mathrm{T}$ & $\mathrm{T}$ & $\mathrm{C} / \mathrm{T}$ & $\mathrm{G} / \mathrm{T}$ \\
\hline S3 & $\mathrm{T} / \mathrm{CR}$ & 1.2 & C & G & $\mathrm{C} / \mathrm{T}$ & C & $G$ & $C$ & $C$ & G & $C$ & G & $\mathrm{T}$ & A & G & $\mathrm{G} / \mathrm{A}$ & $C / A$ & $\mathrm{C} / \mathrm{T}$ & $\mathrm{G} / \mathrm{T}$ & $G$ & G & $\mathrm{C} / \mathrm{T}$ & $\mathrm{T}$ & G & $\mathrm{C} / \mathrm{T}$ & $\mathrm{G} / \mathrm{T}$ \\
\hline S4 & $\mathrm{T} / \mathrm{C}$ & 0.3 & - & G & $C$ & $\mathrm{C} / \mathrm{T}$ & $G$ & $C$ & $C$ & $G$ & $C$ & G & $\mathrm{T}$ & A & G & A & $C / A$ & C & $\mathrm{G} / \mathrm{T}$ & $\mathrm{G}$ & A & C & $\mathrm{T}$ & $\mathrm{G} / \mathrm{T}$ & C & $\mathrm{T}$ \\
\hline S5 & $B / C$ & $U^{a}$ & C & G & C & C & $G$ & $C$ & $C$ & G & $C$ & G & $\mathrm{T}$ & C & C & A & $C / A$ & $\mathrm{C} / \mathrm{T}$ & $\mathrm{G} / \mathrm{T}$ & $G$ & A & C & $\mathrm{T}$ & $\mathrm{T}$ & C & $G$ \\
\hline S6 & $\mathrm{B} / \mathrm{C}$ & 0.3 & C & G & C & C & $G$ & $C$ & $C$ & $G$ & $C$ & G & $\mathrm{T}$ & A & G & A & C & C & $\mathrm{G} / \mathrm{T}$ & G & A & $\mathrm{T}$ & $\mathrm{T}$ & $\mathrm{G} / \mathrm{T}$ & C & $\mathrm{T}$ \\
\hline S7 & $B / C$ & 0.15 & - & G & $C$ & C & $G$ & $C$ & $C$ & $G$ & $C$ & G & $\mathrm{T}$ & C & C & A & $C / A$ & $\mathrm{C} / \mathrm{T}$ & $\mathrm{G} / \mathrm{T}$ & $G$ & A & C & $\mathrm{T}$ & $\mathrm{T}$ & C & $G$ \\
\hline S8 & $B / C$ & U & C & G & C & C & $G$ & $C$ & $C$ & G & $C$ & G & $\mathrm{T}$ & $C / A$ & $\mathrm{G} / \mathrm{C}$ & A & $C / A$ & C & G & $G$ & A & $\mathrm{C} / \mathrm{T}$ & $\mathrm{T}$ & G & C & $\mathrm{G} / \mathrm{T}$ \\
\hline S9 & $B / C$ & 0.5 & C & G & $C$ & C & G & $C$ & $C$ & G & $C$ & G & $\mathrm{T}$ & $C / A$ & $\mathrm{G} / \mathrm{C}$ & A & $C / A$ & $\mathrm{C} / \mathrm{T}$ & G & G & A & C & $\mathrm{T}$ & $\mathrm{T}$ & $\mathrm{C} / \mathrm{T}$ & $\mathrm{G} / \mathrm{T}$ \\
\hline S10 & $B / C$ & 0.1 & C & G & $C$ & C & G & $C$ & $C$ & $G$ & $C$ & G & $\mathrm{T}$ & $C / A$ & $\mathrm{G} / \mathrm{C}$ & A & $C$ & C & G & G & $\mathrm{G} / \mathrm{A}$ & $\mathrm{T}$ & $\mathrm{T}$ & $\mathrm{G} / \mathrm{T}$ & $\mathrm{C} / \mathrm{T}$ & $\mathrm{G} / \mathrm{T}$ \\
\hline S11 & $\mathrm{T} / 2 \mathrm{WW}$ & 0.6 & C & G & $C$ & $\mathrm{C} / \mathrm{T}$ & G & $C$ & $C$ & G & $C$ & G & $T$ & A & G & A & A & C & $\mathrm{G} / \mathrm{T}$ & G & A & C & $\mathrm{T}$ & $\mathrm{T}$ & C & $\mathrm{T}$ \\
\hline S12 & $T / 2 W W$ & 2 & C & G & $C$ & $\mathrm{C} / \mathrm{T}$ & G & $C$ & $c$ & G & $C$ & G & $\mathrm{C} / \mathrm{T}$ & A & G & A & C & C & G & G & A & C & T & $\mathrm{G} / \mathrm{T}$ & $\mathrm{C} / \mathrm{T}$ & $\mathrm{G} / \mathrm{T}$ \\
\hline S13 & $\mathrm{T} / 2 \mathrm{WW}$ & 0.3 & C & G & C & $\mathrm{C} / \mathrm{T}$ & G & C & $C$ & G & $C$ & G & $\mathrm{T}$ & A & G & A & A & C & G & G & A & $C / T$ & $\mathrm{~T}$ & $\mathrm{~T}$ & C & G \\
\hline S14 & $\mathrm{T} / 2 \mathrm{WW}$ & 0.2 & C & G & C & C & G & $C$ & $C$ & G & $C$ & G & $T$ & A & G & A & $C / A$ & C & $\mathrm{T}$ & G & $\mathrm{G} / \mathrm{A}$ & C & $\mathrm{T}$ & G & $\mathrm{C} / \mathrm{T}$ & $\mathrm{T}$ \\
\hline S15 & $\mathrm{T} / 2 \mathrm{WW}$ & 1.2 & C & G & C & C & G & $C$ & C & G & $C$ & G & $\mathrm{T}$ & A & G & A & A & C & $\mathrm{G} / \mathrm{T}$ & G & $\mathrm{G} / \mathrm{A}$ & C & $\mathrm{T}$ & G & C & G \\
\hline S16 & $\mathrm{T} / 2 \mathrm{WW}$ & 0.1 & C & G & C & C & G & $C$ & $C$ & G & $C$ & G & $\mathrm{T}$ & A & G & A & $C / A$ & C & G & G & A & C & $\mathrm{T}$ & $\mathrm{T}$ & C & $\mathrm{T}$ \\
\hline S17 & $\mathrm{T} / 2 \mathrm{WW}$ & 0.8 & C & G & $C$ & C & G & $C$ & $C$ & G & $C$ & G & $\mathrm{T}$ & A & G & A & $C / A$ & C & G & G & A & $C / T$ & $\mathrm{~T}$ & $\mathrm{G} / \mathrm{T}$ & $\mathrm{C} / \mathrm{T}$ & $\mathrm{G} / \mathrm{T}$ \\
\hline S18 & $\mathrm{T} / 2 \mathrm{WW}$ & 2.8 & C & G & C & $\mathrm{C} / \mathrm{T}$ & G & $C$ & $C$ & G & $C$ & G & $\mathrm{T}$ & A & G & A & $C / A$ & C & $\mathrm{G} / \mathrm{T}$ & G & A & C & $\mathrm{C} / \mathrm{T}$ & $\mathrm{G} / \mathrm{T}$ & $\mathrm{C} / \mathrm{T}$ & $\mathrm{G} / \mathrm{T}$ \\
\hline S19 & $\mathrm{T} / 2 \mathrm{WW}$ & 0.8 & C & G & C & C & G & $C$ & $C$ & G & $C$ & G & $\mathrm{T}$ & A & G & A & A & C & G & G & A & C & $\mathrm{T}$ & $\mathrm{T}$ & C & $\mathrm{T}$ \\
\hline S20 & $\mathrm{T} / 2 \mathrm{WW}$ & 0.4 & C & G & $C$ & C & G & $C$ & $C$ & G & $C$ & G & $\mathrm{T}$ & A & G & $\mathrm{G} / \mathrm{A}$ & $C$ & C & $\mathrm{G} / \mathrm{T}$ & G & A & $C / T$ & $\mathrm{~T}$ & $\mathrm{G} / \mathrm{T}$ & $\mathrm{C} / \mathrm{T}$ & $\mathrm{G} / \mathrm{T}$ \\
\hline S21 & $\mathrm{T} / 2 \mathrm{WW}$ & 0.3 & C & G & $C$ & C & G & $C$ & $C$ & G & $C$ & G & $T$ & A & G & A & $C / A$ & C & G & G & A & C & $\mathrm{T}$ & $\mathrm{G} / \mathrm{T}$ & $\mathrm{C} / \mathrm{T}$ & $T$ \\
\hline S22 & $\mathrm{T} / 2 \mathrm{WW}$ & 0.7 & C & G & C & C & G & $C$ & $C$ & G & $C$ & G & $T$ & A & G & G & A & C & G & G & G & C & $\mathrm{C} / \mathrm{T}$ & G & C & $\mathrm{T}$ \\
\hline S23 & $\mathrm{T} / 2 \mathrm{WW}$ & 1.3 & C & G & C & C & G & C & $C$ & G & $C$ & G & $\mathrm{T}$ & A & G & A & $C / A$ & C & $\mathrm{G} / \mathrm{T}$ & G & A & $C / T$ & $\mathrm{~T}$ & $\mathrm{G} / \mathrm{T}$ & C & $\mathrm{T}$ \\
\hline S24 & $\mathrm{T} / \mathrm{X} \|$ & $0.03^{b}$ & C & G & C & C & G & $C$ & $C$ & G & $C$ & $\mathrm{G} / \mathrm{A}$ & $T$ & A & G & A & $C / A$ & C & G & G & A & $\mathrm{C} / \mathrm{T}$ & $T$ & $\mathrm{G} / \mathrm{T}$ & $\mathrm{T}$ & $\mathrm{G} / \mathrm{T}$ \\
\hline S25 & T/XIV & $0.01^{b}$ & - & G & C & C & - & $C$ & $T$ & G & $C$ & - & $\mathrm{T}$ & A & G & A & $C / A$ & C & $\mathrm{T}$ & G & G & C & $\mathrm{T}$ & G & $\mathrm{C} / \mathrm{T}$ & $\mathrm{G} / \mathrm{T}$ \\
\hline S26 & T/XIV & 0.16 & C & G & $C$ & C & G & $C$ & $C$ & G & $C$ & $G / A$ & $\mathrm{~T}$ & A & G & A & $C / A$ & C & $\mathrm{T}$ & G & A & C & $\mathrm{T}$ & $\mathrm{G} / \mathrm{T}$ & C & $\mathrm{G} / \mathrm{T}$ \\
\hline
\end{tabular}

a $\mathrm{U}$ - DNA concentration was not determined due to complete inhibiton of Quantifiler" Human DNA Quantification Kit reaction.

b DNA concentration may be higher as significant inhibition of Quantifilerm Human DNA Quantification Kit reaction was observed. DNA markers are shown in the following order from 1 to 24: 1-N29insA, 2-rs11547464, 3-rs885479, 4-rs1805008, 5-rs1805005, 6-rs1805006, 7-rs1805007, 8-rs1805009, 9-Y152OCH, 10-rs2228479, 11-rs1110400, 12-rs28777, 13- rs 16891982, 14- rs12821256, 15-rs4959270, 16- rs12203592, 17- rs1042602, 18-rs1800407, 19-rs2402130, 20-rs12913832, 21- rs2378249, 22-rs12896399, 23-rs1393350, 24- rs683. Samples S5 and S7 come from the same individual. In the column Sample/postmortem age: T indicates tooth and B indicates bone. CR-indicates contemporary and relatively fresh specimen (one to two years postmortem age); C-indicates contemporary but older than CR; 2WW-indicates samples from the World War 2; XII and XIV-indicate centuries assumed from historical evidence. 
Table 2 HIrisPlex-based eye and hair colour prediction results for the $\mathbf{2 6}$ samples tested
Sample Probability values of Probability values Inferred most Accuracy probability hair colour categories of hair colour shade likely hair colour

Accuracy probability value

Probability values of Inferred most

Accuracy probability value

o a $>300$ European test set

eye colour

colour

a $>3800$ European test set

S1

Brown 0.367
Red 0.002

Dark Brown

$78.5 \%$

Dark 0.732

Black 0.499

Blue 0.317

Brown

$87.5 \%$

Blond 0.133

S2

$$
\text { Brown } 0.246
$$

Red 0.001

Light 0.655

Dark Blond/Brown

Int. 0.193

Brown 0.490

Black 0.326

Dark 0.345

Blond 0.427

S3

Brown 0.496

Red 0.001

Light 0.215

Dark 0.785

Dark Brown

Black 0.406

Blond 0.098

S4

Brown 0.064

Light 0.976

Red 0.048

Dark 0.024

Light Blond

Black 0.025

Blond 0.864

S5

Brown 0.251

Light 0.020

Red 0.000

Dark 0.980

Black 0.729

Blond 0.020

S6

Brown 0.227

Light 0.171

Dark 0.829

Black 0.636

Blond 0.136

S7

Brown 0.282

Red 0.000

Light 0.030

Dark 0.970

Black/Dark Brown

Black 0.690

Blond 0.028

S8

$\begin{array}{cc}\text { Brown } 0.246 & \text { Light } 0.196 \\ \text { Red } 0.000 & \text { Dark } 0.804\end{array}$

Dark 0.804

Dark Brown

Black 0.594

Blond 0.160 
Table 2 HIrisPlex-based eye and hair colour prediction results for the $\mathbf{2 6}$ samples tested (Continued)

S9

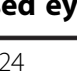

Light 0.212
Dark 0.788

Red 0.001

Black 0.538

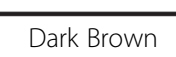

$78.5 \%$

Blue 0.911

Blue

Int. 0.057

Brown 0.032

$\mathrm{S} 10$

Blond 0.136

Brown 0.153

Light 0.016

Black

$87.5 \%$

Blue 0.001

Brown

Int. 0.016

Black 0.829

Dark 0.984

Brown 0.983

Blond 0.017

S11

Brown 0.076

Light 0.964

Light Blond

$69.5 \%$

Blue 0.950

Int. 0.030

Black 0.035

Dark 0.036

Blond 0.852

S12

Brown 0.044

Light 0.969

Light Blond

$69.5 \%$

Brown 0.020

Blue 0.937

Int. 0.041

Brown 0.022

Black 0.049

Blond 0.817

S13

Brown 0.180

Light 0.883

Dark Blond/Light Brown

Dark 0.117

Black 0.119

Blond 0.676

S14

Brown 0.157

Light 0.907

Dark 0.093

Black 0.072

Blond 0.766

S15

Brown 0.168

Red 0.004

Light 0.927

Light Blond

Dark 0.073

Black 0.064

Blond 0.765

S16

$\begin{array}{cc}\text { Brown } 0.089 & \text { Light } 0.951 \\ \text { Red } 0.002 & \text { Dark } 0.049 \\ \text { Black } 0.054 & \\ \text { Blond } 0.856 & \end{array}$

Light Blond

$69.5 \%$

Blue 0.229

Int. 0.128

Brown 0.643

Blue 0.899

Int. 0.066

Brown 0.035

$69.5 \%$

Blue 0.870

Int. 0.076

Brown 0.053

Blue 0.950

Blue

$99 \%$

Int. 0.030

Brown 0.020 
Table 2 HIrisPlex-based eye and hair colour prediction results for the $\mathbf{2 6}$ samples tested (Continued)

S17

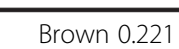

Red 0.00

Black 0.263

Blond 0.514

S18

Brown 0.083

Red 0.090

Black 0.024

Blond 0.802

S19

Brown 0.075

Red 0.002

Black 0.039

Blond 0.883

S20

Brown 0.246

Red 0.001

Black 0.183

Blond 0.570

S21

Brown 0.089

Red 0.002

Black 0.054

Blond 0.856

S22

Brown 0.155

Red 0.008

Black 0.053

Blond 0.783

S23

Brown 0.218

Red 0.002

Black 0.248

Blond 0.532

S24

$\begin{array}{ccc}\text { Brown } 0.202 & \text { Light } 0.695 & \text { Dark Blond/Brown } \\ \text { Red } 0.002 & \text { Dark } 0.305 & \\ \text { Black } 0.301 & \\ \text { Blond } 0.495 & & \end{array}$

$78.5 \%$

Light 0.696

Dark Blond/Brown

Light 0.969

Light Blond

$69.5 \%$

Dark 0.031

Light 0.968

Light Blond

Dark 0.032

Light 0.727

Dark Blond/Brown

Dark 0.273

Light 0.951

Light Blond

Dark 0.049

Light 0.934

Light Blond

Dark 0.066

Light 0.696

Dark Blond/Brown

Dark 0.304

$78.5 \%$

Blue 0.207

Int. 0.161

Brown 0.632

Blue 0.937

Int. 0.041

Brown 0.022

Blue 0.950

Int. 0.030

Brown 0.020

Blue 0.207

Int. 0.161

Brown 0.632

Blue 0.937

Int. 0.041

Brown 0.022

Blue 0.870

Int. 0.076

Brown 0.053

Blue 0.150

Int. 0.140

Brown 0.711

Blue 0.277

Int. 0.179

Brown 0.543

$78.5 \%$
Brown

$91.9 \%$

$91.9 \%$

$97.4 \%$

$95.6 \%$

$94 \%$

$90.4 \%$ 
Table 2 HIrisPlex-based eye and hair colour prediction results for the $\mathbf{2 6}$ samples tested (Continued)

S25

-

S26

$\begin{array}{ccccc}\text { Brown } 0.131 & \text { Light } 0.918 & \text { Light Blond } & 69.5 \% & \text { Blue } 0.919 \\ \text { Red } 0.007 & \text { Dark } 0.082 & & \text { Int. } 0.048 & \text { Blue } \\ \text { Black } 0.078 & & \text { Brown } 0.033 & \end{array}$

Blond 0.784

Probability estimation and final prediction of eye colour was done according to Walsh et al. 2011 [24] and eye colour accuracy estimation according to Walsh et al. 2012 [26].

Probability and accuracy estimation and final prediction for hair colour and hair colour shade was done according to Walsh et al. 2012 [40].

$\begin{array}{lcc}\text { Blue } 0.899 & \text { Blue } & 95.6 \% \\ \text { Int. } 0.066 & & \\ \text { Brown } 0.035 & & 97.4 \% \\ \text { Blue } 0.919 & \text { Blue } & \\ \text { Int. } 0.048 & & \\ \text { Brown } 0.033 & & \end{array}$


$(\mathrm{S} 1=0.490, \mathrm{~S} 2=0.552$ and $\mathrm{S} 3=0.539$, with accuracies $\mathrm{S} 1=87.5 \%, \mathrm{~S} 2=91 \%$, and S3 $=90.4 \%$, Table 2 ). In cases S1 and S3 hair colour was predicted as dark brown (accuracy 78.5\%) and in case S2 hair colour was predicted as dark blond/brown (accuracy 78.5\%, Table 2). Samples S1 and S3 were not associated with any reference material and all the genetic data, including eye and hair colour inference, may be used in further investigating these cases. Sample S3 was associated with a reference sample from a putative close relative but in the course of genetic analysis the assumed hypothesis of a relationship was denied.

Occasionally, human skeletal remains are discovered coincidentally by workers or sightseers. They may be of old or young post mortem age. Sample S4 came from the human remains found in the field near Sieradz in central Poland. Anthropological examination revealed that the bones belong to a woman of about 40 years of age at the time of death. The skeletal remains may have rested in the soil for a very long time as they appeared to be seriously decayed. Two teeth were taken from the maxilla for DNA extraction. A partial NGM STR profile was determined (seven markers were genotyped successfully) and the positive amelogenin result confirmed the female origin suggested from the anthropological investigation. In this case the level of DNA concentration was critical as Quantifiler $^{\text {TM }}$ Human DNA Quantification Kit (Applied Biosystems, Foster City, CA, USA) indicated merely $7 \mathrm{pg} / \mu \mathrm{l}$ of human DNA. As template DNA concentration in the HIrisPlex reaction equalling approximately 30 pg was below the genotyping accuracy threshold of approximately $60 \mathrm{pg}$ as previously established [40], genotyping was repeated three times. Results for all but one (86insC MC1R) HIrisPlex SNPs were obtained successfully. Blue eye colour was inferred with high probability $(P=0.919)$ and accuracy $(P=97.4)$, (Table 2). Blond hair color was inferred with a probability of 0.864 and light hair colour shade with a probability of 0.976 so that light blond is assumed as the most likely hair colour. However, red hair cannot be excluded because of the missing ins86A $M C 1 R$ genotype. Although this DNA variant (indel) is very rare in the general European population (allele observed 4 times among more than 1,000 samples, data not shown), its impact on red hair colour is very strong.

Samples S5 to S10 came from human remains discovered in one of several caves located in the landscape park Dolina Będkowska near Kraków (Poland). Anthropological examination indicated that the revealed 13 skeletal elements, including pelvis, femurs, humerus, ribs, vertebras, mandible comprising two teeth and an incomplete skull, may belong to 6 individuals. The same mtDNA and STR profiles were ascertained in the femur (S5) and the humerus (S7) indicating that these bones likely belong to a single female skeleton (data not shown). Overall genetic analysis showed that in fact all remains came from only five individuals, two females (S5/S7 and S9) and three males (S6, S8 and S10). However, only a partial STR profile was determined in S7 showing more serious degradation compared with S5. Both types of bone (femur and humerus) are usually considered a good source of DNA and thus the observed difference may be coincidental. Complete 24 SNP HIrisPlex profiles were generated for all samples except for S7 where again the ins86A $M C 1 R$ genotype was missing. The obtained data show that from all 24. HIrisPlex polymorphisms indel position 86insA in $M C 1 R$ seems most affected by suboptimal DNA concentration and/or DNA degradation. The S7 profile was also inconsistent among three repeated analyses carried out in terms of rs4959270. From the HIrisPlex profile ascertained in sample S5 we could further conclude that drop-out (rs4959270C/A -> A) occurred in this position in sample S7 (observed twice among three repetitions) keeping with the hypothesis that indeed both samples derive from the same individual. Inferred eye and hair colours for S5 and S7 are blue and black/dark brown, respectively, with highly similar probabilities and accuracies agreeing with the idea that they were derived from the very same individual. Notably, strong PCR inhibition prevented determination of DNA concentration in samples S5 and S8 using Quantifiler ${ }^{\mathrm{TM}}$ Human DNA Quantification Kit (Table 1). Nevertheless, genotyping was possible in both samples showing that the HIrisPlex assay is indeed insensible to inhibition similarly to commercial STR kits used for human identification purposes. Inferred eye colour was blue for S9 (probability 0.911, accuracy 97.4\%) and brown for S6, S8 and S10 (probabilities $0.972,0.892$, and 0.983 , respectively; accuracies 99\%, 95.6\% and 99\%, respectively). Inferred hair colour was dark brown for S6, S8 and S9 (accuracy 78.5\%) and black for S10 (accuracy of $87.5 \%$ ).

\section{Samples from World War II}

DNA analysis from skeletal remains may help to solve various controversies concerning historical figures. As an illustrative example, we analysed tooth S11 (Figure 1) collected from the corpse of General Władysław Sikorski. During World War II, Sikorski was Commander-in-Chief of the Polish Armed Forces and also was Prime Minister of the Polish government in exile. Sikorski died in an airplane crash at Gibraltar in 1943. His body was buried in a cemetery in Newark (UK) and after exhumation in 1993 was finally placed in the crypt of the cathedral of the Royal Castle on Wawel Hill in Kraków (Poland). Since the hypothesis that an accident had caused the airplane crush at Gibraltar was questioned, in 2008 Władysław Sikorski was again exhumed and the body was thoroughly examined [41]. Previous mitochondrial DNA analysis of the 
tooth was crucial for identification of General Sikorski's remains, but a complete Identifiler STR profile and a complete Yfiler Y-STR profile were obtained too, indicating reasonably good DNA preservation [42]. A complete HIrisPlex SNP profile was generated and is shown in Figure 2. The HIrisPlex model predicted blue eye colour with high probability $(P=0.950)$ and high accuracy $(99 \%)$ as well as blond hair colour $(P=0.852)$ and a light hair colour shade $(P=0.964)$ so that light blond can be assumed as the most likely hair colour (accuracy 69.5\%). It is worth noting that in this particular case the eye/hair colour predictions could be confirmed with known data about Władysław Sikorski's appearance. Although colour photographs of the General were unavailable and all known colour portraits were painted many years after his death and thus may not be considered most reliable, the fact that Władysław Sikorski indeed had blue eyes and blond hair, as predicted via HIrisPlex, was obtained from historical scripts [43].

DNA analysis of recent human skeletal remains can also be helpful in the identification of war victims. As examples, teeth S12 to S23 were collected from persons who were killed during World War II in a prison located in Poznań (Poland). In 1942, 14 skulls were sent to the Natural History Museum in Vienna and at that time they were exhibited as examples of "sub-human" skulls. In 1998, these skulls were returned to Poland, and the Institute of National Remembrance ordered examination of these human remains. In all 12 cases, besides mitochondrial DNA data, complete Minifiler STR profiles and partial Yfiler Y-STR profiles were obtained (data not shown) indicating some level of genomic DNA fragmentation. All 12 skulls were included in this study for teeth sampling. In all 12 cases, complete HIrisPlex SNP profiles were generated (Table 1) and the eye and hair colour prediction results are presented in Table 2 . Among these 12 individuals, blue (probabilities 0.87 to 0.95 ) and brown eye colours (probabilities 0.632 to 0.711 ) were inferred with high accuracies (91.9\% to 99\%) as well as light blond, light blond/dark blond and dark

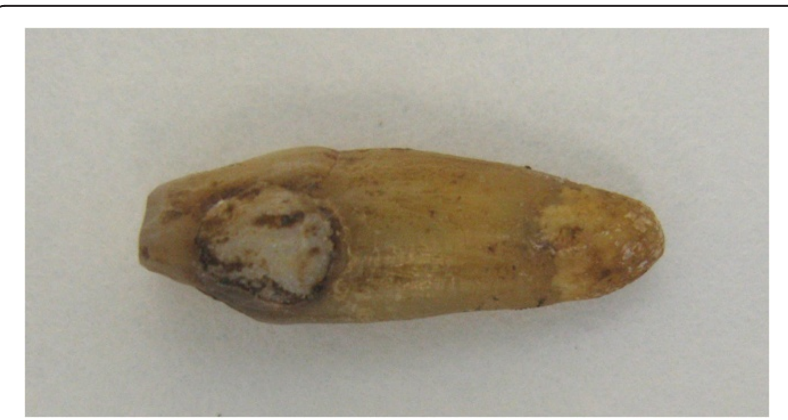

Figure 1 The photograph of the analysed tooth of General Władysław Sikorski (World War II). blond/brown hair colours (accuracies 69.5 to $78.5 \%$ ), as may be expected from individuals of (most likely) Polish European ancestry.

\section{Medieval samples}

Analysis of DNA extracted from old bone samples is often a very challenging task due to very low amounts of DNA achieved, which additionally is very often subject to heavy degradation. Sample S24 represents a controversial case from the Benedictine Abbey in Tyniec near Kraków. During the work undertaken in the crypt of the St. Peter and Paul church belonging to the Abbey, 17 skeletons of alleged abbots were found. The burial was dated to the period of the $12^{\text {th }}$ to $14^{\text {th }}$ centuries. Unexpectedly, the anthropological examination revealed that two skeletons may be of female origin, which indeed was confirmed by DNA analysis (data not shown), while only male monks were expected. One of the two DNA samples was sufficiently preserved to enable analysis of other nuclear markers (data not shown) and was used here for HIrisPlex analysis. The mysterious woman was predicted to have dark blond/brown hair (accuracy of 78.5\%) and brown eyes (accuracy of 90.4\%), (Table 2 and Figure 2B). The somewhat stronger peak imbalance relative to the sample shown in the electropherogram panel A can be explained by the lower DNA quality of this medieval sample and resulting preferential amplification. Consequent higher signals observed at rs1805009 (above 8,000 RFU) led to elevated pull-up peaks. These artifacts, however, did not interfere with the phenotype interpretation from the obtained genotype data.

Samples S25 and S26 came from two skeletons revealed during conservation work conducted in the Church of St. Andrew in Kraków in 2011. The church of St. Andrew was built between 1079 and 1098, and represents a great example of the Romanesque style. Two medieval skeletons were found under the floor between the chancel and the nave of the church. Based on historical markers the grave was dated to originate from the 14th century. Further anthropological examinations indicated that the S25 male died at the approximate age of 60 , whereas the S26 male was approximately 75 years old at the time of death. It is alleged that the skeletons belong to members of the Tęczyński family, representing noble Polish magnates of medieval times. The tooth collected from the deeper burial (S25) was found to be seriously affected by decay, which was reflected by a very low DNA concentration $(3 \mathrm{pg} / \mu \mathrm{l})$ and incomplete autosomal and Y chromosome STR profiles (NGM and Yfiler). Complete mtDNA HVI and HVII profiles were generated in both teeth (data not shown). From these data it was possible to conclude that both skeletons are of male origin and are unrelated in both maternal and paternal lines. From the partial HIrisPlex profile 


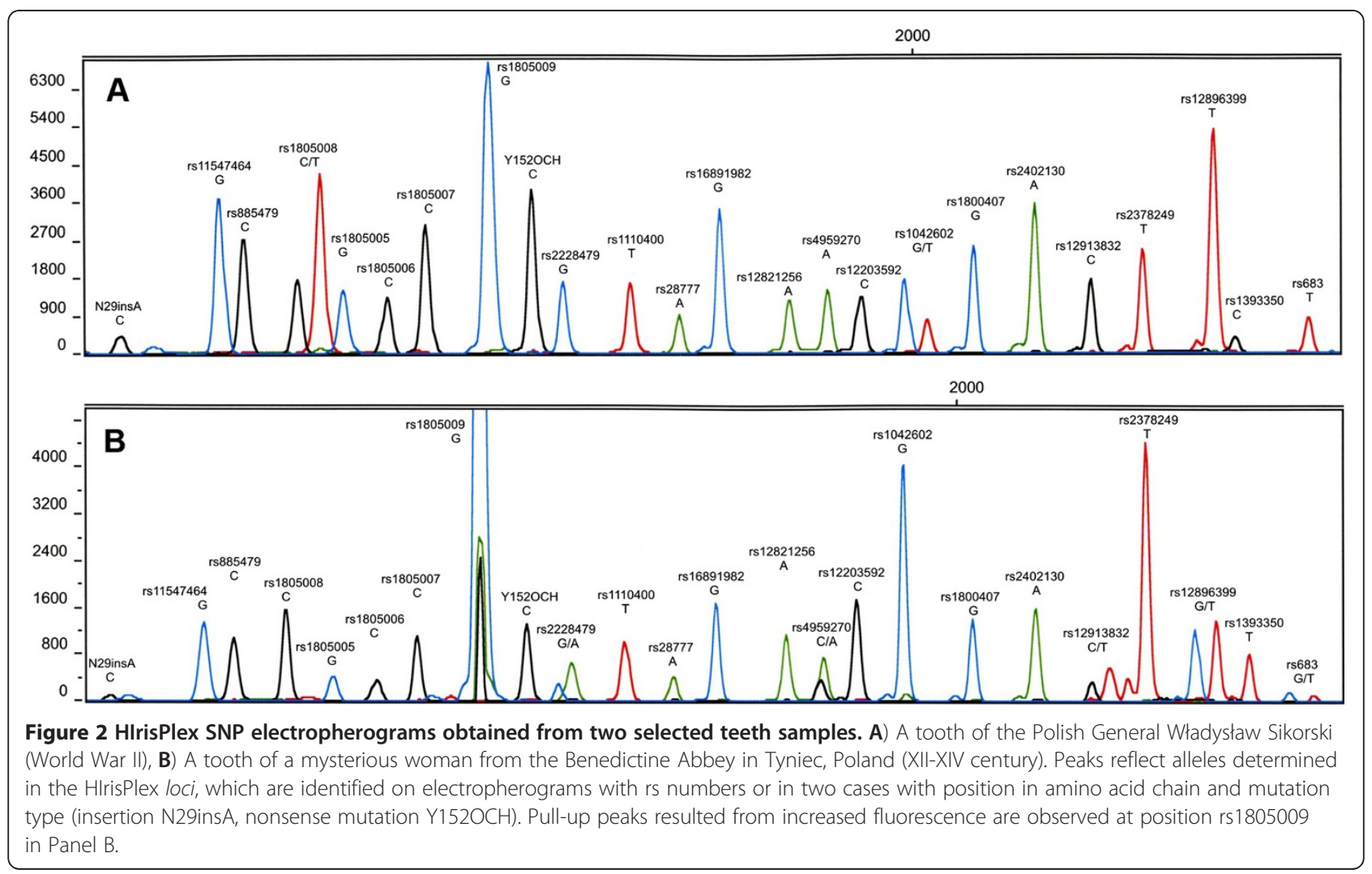

ascertained from S25 we successfully inferred blue eye colour $(P=0.899$, accuracy of $95.6 \%)$, but hair colour could not be inferred because of missing genotypes at three DNA variants (N29insA, rs1805005, rs2228479). The sample S26 revealed a prediction of blond hair colour $(P=0.784)$ together with a light hair colour shade $(\mathrm{P}=0.918)$ concluding that the individual had light blond hair (accuracy of 69.5\%). Eye colour prediction of S26 revealed blue eyes $(\mathrm{P}=0.919$, accuracy of $97.4 \%)$ (Table 2 ).

The HIrisPlex system was initially designed to enable degraded DNA analysis with considering short amplicons (less than $160 \mathrm{bp}$ for all amplicons) [40]. Here we showed that HIrisPlex performs successfully in degraded DNA from skeletal remains of various ages and storage conditions. However, also under such design, the possibility of allelic drop-outs and drop-ins, which have been described as typical phenomenon associated with analysis of low template DNA samples, cannot be eliminated completely. Allele drop-outs, which are explained by stochastic effects, may lead to false homozygote genotypes whereas allele drop-ins are explained by minute contaminations. Both effects may affect final results of DNA-based human identification $[44,45]$. Allelic drop-outs and drop-ins can also have a practical impact on EVC prediction, for example, HIrisPlex-based eye and hair colour prediction. However, the final effect depends on the particular SNP involved, as different SNPs have different impact on the final eye and hair colour prediction. Such influence may be particularly strong for strong DNA predictors, such as the IrisPlex/ HIrisPlex marker rs12913832 in the HERC2 gene, which strongly determines blue/brown eye colour. From the homozygote CC/GG genotype blue eye colour is predicted while the heterozygote CT/GA genotype most often indicate hazel/light brown eye colour [26]. A similar situation occurs in case of several DNA variants in the MC1R gene, such as N29insA, rs1805007 and rs1805008 that have high penetrance among red hair colour individuals. All samples analysed here were genotyped at least twice and in most cases the results were consistent. Inconsistencies were observed mainly at two DNA variants, the insertion/deletion polymorphism N29insA (first position in the assay) and the polymorphism C/A rs4959270 (15. position in the assay). N29insA was most sensitive to suboptimal quality/ quantity of template DNA. The peak height is generally lower compared to the other SNPs in the HIrisPlex assay and did not reach detection limit settled at 50 RFU five times in the course of repeated analyses. It should be also mentioned that in three cases (S4, S7 and S25) this polymorphism remained undetermined (Table 1). For rs4959270, we most probably observed drop-out in two samples (S14 and S17). Table 2 shows the prediction results under the assumption of the presence of the heterozygous state CA/GT in both cases as is presented in Table 1. This discrepancy has little influence on the 
prediction values but affects the conclusion for S14 in terms of hair colour (light blond/dark blond for homozygote $\mathrm{C}$ vs. light blond for heterozygote $\mathrm{C} / \mathrm{A}$ ). Although drop-out was assumed as more probable, drop-in cannot be completely ruled out. Notably, these two DNA variants were also discussed by Walsh et al. as more sensitive than all other HIrisPlex SNPs to template DNA quantity [40]. In one of the three genotypings performed on sample S13 drop-ins were also identified at rs2402130G (position 19 of the assay), rs12896399 G (position 22) and rs683T (position 24). We assumed that these signals reflect minor contamination because they were detected only once and at significantly lower levels than other peaks in the HIrisPlex profile of this sample. Negative extraction and PCR controls were clean in case of STR analyses in this sample while in some HIrisPlex negative controls one to three peaks at a very low signal level were detected. This effect seems to be stochastic and there is no particular DNA variant in the HIrisPlex assay which can be pointed to as more prone to such minor contamination.

The results obtained here provide further evidence that quality of DNA templates from bone material depends not only on the storage time but more so on environmental conditions affecting the decomposition of the remains. Low temperature and low humidity are known to prevent DNA degradation [46], allowing successful DNA analysis even after tens of thousands of years [1]. Here we show that a complete 24 SNP HIrisPlex profile was obtained from a $12^{\text {th }}$ century sample (S24) collected from a skeleton that rested in relatively favourable conditions in a church. On the other hand, a partial profile was obtained from a relatively young contemporary sample (S17) that was found in open soil. However, it is difficult, as had been outlined previously [46], to draw generalizing conclusions from such data. For instance, a different $12^{\text {th }}$ century sample stored under expectedly similar conditions also excavated from inside a church (S25) yielded a partial HIrisPlex profile.

Obviously, special care is needed when EVC prediction is performed for samples containing DNA in suboptimal quantity and/or quality. Previous preliminary sensitivity testing of the HIrisPlex assay [40] provided an approximate threshold of $60 \mathrm{pg}$, above which allelic drop-out and drop-in were not observed. In the present study, two samples were analysed from an estimated starting template DNA amount of less than $60 \mathrm{pg}$, of which one (S24 approximately $30 \mathrm{pg}$ ) revealed a full HIrisPlex profile allowing both eye and hair colour prediction, while the other (S25 approximately $10 \mathrm{pg}$ ) lacked genotypes at three DNA variants, which, because of the particular DNA variants involved in the drop-out, did allow for eye colour, but not hair colour prediction. However, the concentration estimates established for these two samples may not be realistic as both extracts displayed signs of
DNA inhibition from Quantifiler RT-PCR measurement. In any case, application of the HIrisPlex assay from low quality/quantity DNA samples, such as DNA extracted from skeletal remains or touched objects, should be accompanied with appropriate rigor, meeting recommendations settled for low template DNA samples [47].

\section{Conclusions}

It can be anticipated that DNA prediction of EVC will soon become more widely used in genetic studies of human remains in evolutionary, anthropological and forensic investigations. The recently introduced HIrisPlex system [40] provides a convenient molecular tool for simultaneous prediction of eye and hair colour categories from DNA. As demonstrated here, the HIrisPlex system is sufficiently sensitive and robust to enable successful analysis of bones and teeth of various ages, including medieval (and expectedly older) samples. HIrisPlex can be successfully implemented in a forensic DNA laboratory equipped with the standard layout for DNA identity testing and specialized on DNA extraction from skeletal remains, and likewise in any dedicated ancient DNA laboratory. With the current study, we aim to encourage the anthropological and forensic genetic community to use the HIrisPlex system for DNA-based eye and hair colour prediction in future evolutionary, anthropological studies and for forensic case work involving skeletal remains.

\section{Abbreviations}

aDNA: Ancient DNA; DTT: Dithiothreitol; DVI: Disaster victim identification; EDTA: Ethylenediaminetetraacetic acid; EVC: Externally visible characteristic; FDP: Forensic DNA phenotyping; HGDP-CEPH: Human genome diversity cell line panel Foundation Jean Dausset-CEPH Paris; indel: Insertion-deletion polymorphism; mtDNA: Mitochondrial DNA; NGS: Next generation DNA sequencing; RT-PCR: Real time polymerase chain reaction; SBE: Single-base extension; SDS: Sodium dodecyl sulfate; SNP: Single nucleotide polymorphism; STR: Short tandem repeat (polymorphism); WGS: Whole genome sequencing; Y-STR: Y chromosome STR.

\section{Competing interests}

The authors declare that they have no competing interests.

\section{Authors' contributions}

WB and MK conceived and designed the study, provided resources, interpreted results and wrote the manuscript. JDB carried out the genotyping and elaborated results. EP participated in genotyping data analysis. SW carried out the prediction of phenotypes from genotypes and helped in manuscript writing. TK and HG provided the samples and helped to draft the case description parts of the manuscript. All authors read and approved the final manuscript.

\section{Acknowledgments}

We wish to thank all colleagues who helped with sample selection, technical analyses and provided anthropological information, especially Andrzej Sekuła, Agnieszka Parys-Proszek and Andrzej Czubak. This study was supported in part by a grant from the Ministry of Science and Higher Education in Poland no ON301115136 to WB and in part by the Netherlands Forensic Institute (NFI) and by a grant from the Netherlands Genomics Initiative (NGI)/ Netherlands Organization for Scientific Research (NWO) within the framework of the Forensic Genomics Consortium Netherlands (FGCN). EP and WB have received funding support from the European Union Seventh Framework Programme (FP7/2007-2013) under grant agreement no 285487. 


\section{Author details}

${ }^{1}$ Institute of Forensic Research, Section of Forensic Genetics, Kraków, Poland. ${ }^{2}$ Department of Forensic Molecular Biology, Erasmus MC University Medical Center Rotterdam, Rotterdam, The Netherlands. ${ }^{3}$ Department of Anthropology, Institute of Zoology, Faculty of Biology and Earth Sciences, Jagiellonian University, Kraków, Poland. ${ }^{4}$ Department of Genetics and Evolution, Institute of Zoology, Faculty of Biology and Earth Sciences, Jagiellonian University, Kraków, Poland.

Received: 2 August 2012 Accepted: 14 November 2012

Published: 14 January 2013

\section{References}

1. Green RE, Krause J, Briggs AW, Maricic T, Stenzel U, Kircher M, Patterson N, Li H, Zhai W, Fritz MH, Hansen NF, Durand EY, Malaspinas AS, Jensen JD, Marques-Bonet T, Alkan C, Prüfer K, Meyer M, Burbano HA, Good JM, Schultz R, Aximu-Petri A, Butthof A, Höber B, Höffner B, Siegemund M, Weihmann A, Nusbaum C, Lander ES, Russ C, Novod N, et al: A draft sequence of the Neandertal genome. Science 2010, 328:710-722

2. Reich D, Green RE, Kircher M, Krause J, Patterson N, Durand EY, Viola B, Briggs AW, Stenzel U, Johnson PL, Maricic T, Good JM, Marques-Bonet T, Alkan C, Fu Q, Mallick S, Li H, Meyer M, Eichler EE, Stoneking M, Richards M, Talamo S, Shunkov MV, Derevianko AP, Hublin JJ, Kelso J, Slatkin M, Pääbo S: Genetic history of an archaic hominin group from Denisova Cave in Siberia. Nature 2010, 468:1053-1060.

3. Reich D, Patterson N, Kircher M, Delfin F, Nandineni MR, Pugach I, Ko AM, Ko YC, Jinam TA, Phipps ME, Saitou N, Wollstein A, Kayser M, Pääbo S, Stoneking M: Denisova admixture and the first modern human dispersals into Southeast Asia and Oceania. Am J Hum Genet 2011, 89:516-528.

4. Skoglund P, Jakobsson M: Archaic human ancestry in East Asia. Proc Natl Acad Sci U S A 2011, 108:18301-18306.

5. Brenner $\mathrm{CH}$, Weir BS: Issues and strategies in the DNA identification of World Trade Center victims. Theor Popul Biol 2003, 63:173-178.

6. Prinz M, Carracedo A, Mayr WR, Morling N, Parsons TJ, Sajantila A, Scheithauer R, Schmitter H, Schneider PM: International society for forensic genetics. DNA commission of the international society for forensic genetics (ISFG): recommendations regarding the role of forensic genetics for disaster victim identification (DVI). Forensic Sci Int Genet 2007, 1:3-12.

7. Buckleton JS, Krawczak M, Weir BS: The interpretation of lineage markers in forensic DNA testing. Forensic Sci Int Genet 2011, 5:78-83.

8. Gill P, Ivanov PL, Kimpton C, Piercy R, Benson N, Tully G, Evett I, Hagelberg E, Sullivan K: Identification of the remains of the Romanov family by DNA analysis. Nat Genet 1994, 6:130-135.

9. Ivanov PL, Wadhams MJ, Roby RK, Holland MM, Weedn WW, Parsons TJ: Mitochondrial DNA sequence heteroplasmy in the Grand Duke of Russia Georgij Romanov establishes the authenticity of the remains of Tsar Nicholas II. Nat Genet 1996, 12:417-420

10. Coble MD, Loreille OM, Wadhams MJ, Edson SM, Maynard K, Meyer CE, Niederstätter $H$, Berger C, Berger B, Falsetti AB, Gill P, Parson W, Finelli LN: Mystery solved: the identification of the two missing Romanov children using DNA analysis. PLoS One 2009, 4:4838.

11. Coble MD: The identification of the Romanovs: can we (finally) put the controversies to rest? Investig Genet 2011, 2:20.

12. Bogdanowicz W, Allen M, Branicki W, Lembring M, Gajewska M, Kupiec T: Genetic identification of putative remains of the famous astronomer Nicolaus Copernicus. Proc Natl Acad Sci U S A 2009, 106:12279-12282.

13. Caramelli D, Lalueza-Fox C, Capelli C, Lari M, Sampietro ML, Gigli E, Milani L, Pilli E, Guimaraes S, Chiarelli B, Marin VT, Casoli A, Stanyon R, Bertranpetit J, Barbujani G: Genetic analysis of the skeletal remains attributed to Francesco Petrarca. Forensic Sci Int 2007, 173:36-40.

14. Vernesi C, Di Benedetto G, Caramelli D, Secchieri E, Simoni L, Katti E, Malaspina P, Novelletto A, Marin VT, Barbujani G: Genetic characterization of the body attributed to the evangelist Luke. Proc Natl Acad Sci U S A 2001, 98:13460-13463.

15. Kayser M, Schneider PM: DNA-based prediction of human externally visible characteristics in forensics: motivations, scientific challenges, and ethical considerations. Forensic Sci Int Genet 2009, 3:154-161.

16. Kayser $M$, de Knijff $P$ : Improving human forensics through advances in genetics, genomics and molecular biology. Nat Rev Genet 2011, 12:179-192.
17. Valverde P, Healy E, Jackson I, Rees JL, Thody AJ: Variants of the melanocyte-stimulating hormone receptor gene are associated with red hair and fair skin in humans. Nat Genet 1995, 11:328-330.

18. Sulem P, Gudbjartsson DF, Stacey SN, Helgason A, Rafnar T, Magnusson KP, Manolescu A, Karason A, Palsson A, Thorleifsson G, Jakobsdottir M, Steinberg S, Pálsson S, Jonasson F, Sigurgeirsson B, Thorisdottir K, Ragnarsson R, Benediktsdottir KR, Aben KK, Kiemeney LA, Olafsson JH, Gulcher J, Kong A, Thorsteinsdottir U, Stefansson K: Genetic determinants of hair, eye and skin pigmentation in Europeans. Nat Genet 2007, 39:1443-1452

19. Sulem P, Gudbjartsson DF, Stacey SN, Helgason A, Rafnar T, Jakobsdottir M, Steinberg S, Gudjonsson SA, Palsson A, Thorleifsson G, Pálsson S, Sigurgeirsson B, Thorisdottir K, Ragnarsson R, Benediktsdottir KR, Aben KK, Vermeulen SH, Goldstein AM, Tucker MA, Kiemeney LA, Olafsson JH, Gulcher J, Kong A, Thorsteinsdottir U, Stefansson K: Two newly identified genetic determinants of pigmentation in Europeans. Nat Genet 2008, 40:835-837.

20. Sturm RA, Duffy DL, Zhao ZZ, Leite FP, Stark MS, Hayward NK, Martin NG, Montgomery GW: A single SNP in an evolutionary conserved region within intron 86 of the HERC2 gene determines human blue-brown eye color. Am J Hum Genet 2008, 82:424-431.

21. Kayser M, Liu F, Janssens AC, Rivadeneira F, Lao O, van Duijn K, Vermeulen M, Arp P, Jhamai MM, van ljcken WF, den Dunnen JT, Heath S, Zelenika D, Despriet DD, Klaver CC, Vingerling JR, de Jong PT, Hofman A, Aulchenko YS, Uitterlinden AG, Oostra BA, van Duijn CM: Three genome-wide association studies and a linkage analysis identify HERC2 as a human iris color gene. Am J Hum Genet 2008, 82:411-423.

22. Eiberg H, Troelsen J, Nielsen M, Mikkelsen A, Mengel-From J, Kjaer KW Hansen L: Blue eye color in humans may be caused by a perfectly associated founder mutation in a regulatory element located within the HERC2 gene inhibiting OCA2 expression. Hum Genet 2008, 123:177-187.

23. Liu F, van Duijn $K$, Vingerling JR, Hofman A, Uitterlinden AG, Janssens AC, Kayser M: Eye color and the prediction of complex phenotypes from genotypes. Curr Biol 2009, 19:192-193.

24. Walsh S, Liu F, Ballantyne KN, van Oven M, Lao O, Kayser M: IrisPlex: a sensitive DNA tool for accurate prediction of blue and brown eye colour in the absence of ancestry information. Forensic Sci Int Genet 2011, 5:170-180.

25. Walsh S, Lindenbergh A, Zuniga SB, Sijen T, de Knijff P, Kayser M, Ballantyne $\mathrm{KN}$ : Developmental validation of the IrisPlex system: determination of blue and brown iris colour for forensic intelligence. Forensic Sci Int Genet 2011, 5:464-471

26. Walsh S, Wollstein A, Liu F, Chakravarthy U, Rahu M, Seland JH, Soubrane G, Tomazzoli L, Topouzis F, Vingerling JR, Vioque J, Fletcher AE, Ballantyne KN, Kayser M: DNA-based eye colour prediction across Europe with the IrisPlex system. Forensic Sci Int Genet 2012, 6:330-340.

27. Branicki W, Liu F, van Duijn K, Draus-Barini J, Pośpiech E, Walsh S, Kupiec T, Wojas-Pelc A, Kayser M: Model-based prediction of human hair color using DNA variants. Hum Genet 2011, 129:443-454.

28. Branicki W, Brudnik U, Wojas-Pelc AL: Interactions between HERC2, OCA2 and MC1R may influence human pigmentation phenotype. Ann Hum Genet 2009, 73:160-170.

29. Branicki W, Brudnik U, Kupiec T, Wolañska-Nowak P, Wojas-Pelc A: Determination of phenotype associated SNPs in the MC1R gene. $J$ Forensic Sci 2007, 52:349-354.

30. Mengel-From J, Wong TH, Morling N, Rees JL, Jackson IJ: Genetic determinants of hair and eye colours in the Scottish and Danish populations. BMC Genet 2009, 10:88.

31. Mengel-From J, Børsting C, Sanchez JJ, Eiberg H, Morling N: Human eye colour and HERC2, OCA2 and MATP. Forensic Sci Int Genet 2010, 4:323-328.

32. Spichenok O, Budimlija ZM, Mitchell AA, Jenny A, Kovacevic L, Marjanovic D, Caragine T, Prinz M, Wurmbach E: Prediction of eye and skin color in diverse populations using seven SNPs. Forensic Sci Int Genet 2011, 5:472-478.

33. Ruiz Y, Phillips C, Gomez-Tato A, Alvarez-Dios J, Casares de Cal M, Cruz R, Maroñas O, Söchtig J, Fondevila M, Rodriguez-Cid MJ, Carracedo A, Lareu MV: Further development of forensic eye color predictive tests. Forensic Sci Int Genet 2013, 7:28-40.

34. Lalueza-Fox C, Römpler H, Caramelli D, Stäubert C, Catalano G, Hughes D, Rohland N, Pilli E, Longo L, Condemi S, de la Rasilla M, Fortea J, Rosas A, Stoneking M, Schöneberg T, Bertranpetit J, Hofreiter M: A melanocortin 1 receptor allele suggests varying pigmentation among Neanderthals. Science 2007, 318:1453-1455. 
36. Cerqueira CC, Paixão-Côrtes VR, Zambra FM, Salzano FM, Hünemeier T, Bortolini MC: Predicting homo pigmentation phenotype through genomic data: from Neanderthal to James Watson. Am J Hum Biol 2012, 24:705-709.

37. Rasmussen M, Li Y, Lindgreen S, Pedersen JS, Albrechtsen A, Moltke I, Metspalu M, Metspalu E, Kivisild T, Gupta R, Bertalan M, Nielsen K, Gilbert MT, Wang Y, Raghavan M, Campos PF, Kamp HM, Wilson AS, Gledhill A, Tridico S, Bunce M, Lorenzen ED, Binladen J, Guo X, Zhao J, Zhang X, Zhang $H$, Li Z, Chen M, Orlando L, et al: Ancient human genome sequence of an extinct Palaeo-Eskimo. Nature 2010, 463:757-762.

38. Keller A, Graefen A, Ball M, Matzas M, Boisguerin V, Maixner F, Leidinger $P$, Backes C, Khairat R, Forster M, Stade B, Franke A, Mayer J, Spangler J, McLaughlin S, Shah M, Lee C, Harkins TT, Sartori A, Moreno-Estrada A, Henn B, Sikora M, Semino O, Chiaroni J, Rootsi S, Myres NM, Cabrera VM, Underhill PA, Bustamante CD, Vigl EE, et al: New insights into the Tyrolean Iceman's origin and phenotype as inferred by whole-genome sequencing. Nat Commun 2012, 3:698.

39. Bouakaze C, Keyser C, Crubézy E, Montagnon D, Ludes B: Pigment phenotype and biogeographical ancestry from ancient skeletal remains: inferences from multiplexed autosomal SNP analysis. Int J Legal Med 2009, 123:315-325.

40. Walsh S, Liu F, Wollstein A, Kovatsi L, Ralf A, Kosiniak-Kamysz A, Branicki W, Kayser M: The HIrisPlex system for simultaneous prediction of hair and eye colour from DNA. Forensic Sci Int Genet 2013, 7:98-115.

41. Konopka T, Gross A, Woźniak K, Kłys M: Forensic medical examination of the corpse of general Władysław Sikorski, a putative victim of assassination in 1943. Forensic Sci Int 2010, 202:29-33.

42. Kupiec T, Branicki W: Genetic analysis of the putative remains of general Władysław Sikorski. Arch Med Sadowej Kryminol 2009, 59:9-14.

43. Stoma L: Poczet polityków polskich. Warszawa: Oficyna Wydawnicza Grafpunkt; 2000.

44. Gill P, Whitaker J, Flaxman C, Brown N, Buckleton J: An investigation of the rigor of interpretation rules for STRs derived from less than $100 \mathrm{pg}$ of DNA. Forensic Sci Int 2000, 112:17-40.

45. Dixon LA, Murray CM, Archer EJ, Dobbins AE, Koumi P, Gill P: Validation of a 21-locus autosomal SNP multiplex for forensic identification purposes. Forensic Sci Int 2005, 154:62-77.

46. Burger J, Hummel S, Hermann B, Henke W: DNA preservation: a microsatellite-DNA study on ancient skeletal remains. Electrophoresis 1999, 20:1722-1728.

47. Balding DJ, Buckleton J: Interpreting low template DNA profiles. Forensic Sci Int Genet 2009, 4:1-10.

doi:10.1186/2041-2223-4-3

Cite this article as: Draus-Barini et al.: Bona fide colour: DNA prediction of human eye and hair colour from ancient and contemporary skeletal remains. Investigative Genetics 2013 4:3.

\section{Submit your next manuscript to BioMed Central and take full advantage of:}

- Convenient online submission

- Thorough peer review

- No space constraints or color figure charges

- Immediate publication on acceptance

- Inclusion in PubMed, CAS, Scopus and Google Scholar

- Research which is freely available for redistribution 